\title{
Controlling Laminate Plate Elastic Behavior
}

T. Mareš

This paper aims to express the relation of a measure of laminate plate stiffness with respect to the fiber orientation of its plies. The inverse of the scalar product of the lateral displacement of the central plane and lateral loading of the plate is the measure of laminate plate stiffness. In the case of a simply supported rectangular laminate plate this measure of stiffness is maximized, and the optimum orientation of its plies is searched.

Keywords: mechanical properties, laminate, plate, composites.

\section{Introduction}

Problems of structural optimization and analysis of stress and strain often lead to the function optimization problem of minimizing a given functional subject to a system of differential equations and inequalities with some boundary conditions, and a system of integral equations and inequalities. This class of structural optimization problems is called function structural optimization. The branch of mathematics that deals with this problem is called Variational Calculus. The difficulty of solving this problem has led to the idea of transforming the given function optimization problem with the Galerkin method (based on the finite element method) to a problem of parameter optimization. This paper deals with both function optimization and parameter optimization of laminate plate stiffness.

The paper aims to express the relation of a measure of laminate plate stiffness with respect to the fiber orientation of its plies. The inverse of the scalar product of the lateral displacement of the central plane and the lateral loading of the plate is the measure of laminate plate stiffness. In the case of a simply supported rectangular laminate plate this measure of stiffness is maximized, and the optimum orientation of its plies is searched.

We contemplate the problem of rectangular laminate plate with common but fixed given dimensions, number of plies, thickness of plies, mechanical properties of orthotropic plies and lateral loading that is simply suported on its boundary with the aim to specify an orientation of its plies that maximizes the measure of stiffness, which is the inverse of the measure of compliance. The measure of compliance is given by the scalar product of the deflection function describing the deformed middle plane of the plate and the lateral loading over the projection of the plate into the middle plane.

We must search an actual deformed state with respect to the common orientation of the plies. This solution is used in the above expressed measure of compliance, and the minimum of this expression is searched.

\section{Preliminaries}

We contemplate the problem of a rectangular laminate plate with common but given

- dimensions

- number of plies
- thickness of plies

- mechanical properties of orthotropic plies

- lateral loading

that is simply suported on its boundary with the aim of specifying an orientation of its plies that maximizes the measure of stiffness

$$
\mathfrak{B}(w)=\frac{1}{\mathfrak{l}(w)}
$$

where

$$
\mathfrak{l}(w)=\int_{\omega} w(x, y) q(x, y) \mathrm{d} x \mathrm{~d} y
$$

is the measure of compliance and where $w(x, y)$ is the deflection function describing the deformed middle plane of the plate, $q(x, y)$ is the lateral loading, and $\omega$ is the projection of the plate into middle plane $x-y$.

We must search an actual deformed state $w(\boldsymbol{\alpha})$ with respect to the common orientationof the plies $\alpha$. This solution is used in the above expressed measure of compliance, and the minimum of this expression is searched; i.e. we must solve the problem

$$
\alpha=\arg \min _{\alpha} l(w(\alpha))
$$

\section{Common rules}

It is well-known that an actual deformed state minimizes the potential energy

$$
\Pi(\boldsymbol{u})=a(\boldsymbol{u}, \boldsymbol{u})-l(\boldsymbol{u}),
$$

where $a(\boldsymbol{u}, \boldsymbol{u})$ is elastic potential energy

$$
a(\boldsymbol{u}, \boldsymbol{u})=\frac{1}{2} \int_{\Omega} E_{i j k l}(\boldsymbol{x}) \cdot \varepsilon_{i j}(\boldsymbol{u}(\boldsymbol{x})) \cdot \varepsilon_{k l}(\boldsymbol{u}(\boldsymbol{x})) \mathrm{d} \boldsymbol{\Omega}
$$

and $l(\boldsymbol{u})$ is potential energy of external loads

$$
l(\boldsymbol{u})=\int_{\Omega} p_{i} u_{i} \mathrm{~d} \Omega+\int_{\partial \Omega_{t}} t_{i} u_{i} \mathrm{~d} \boldsymbol{\Gamma} .
$$

It is also well-known that in the actual state $\hat{\boldsymbol{u}}$ the potential energy $\Pi$ has the value

$$
\Pi(\hat{\boldsymbol{u}})=-\frac{1}{2} l(\hat{\boldsymbol{u}})<0 .
$$

Hence the problem of maximizing the stiffness measure is transformed into the problem of searching a min-max point of the potential energy

$$
\{\hat{\boldsymbol{E}}, \hat{\boldsymbol{u}}\}=\arg \max _{\boldsymbol{E} \in \mathrm{E}} \min _{\boldsymbol{u} \in \mathbb{U}}(a(\boldsymbol{u}, \boldsymbol{u})-l(\boldsymbol{u})) .
$$




\section{Formulation of the problem}

In the case introduced above there is the problem $\{\hat{w}, \hat{\alpha}\}=\arg \max _{\alpha \in \mathbb{A}} \min _{w \in \mathbb{W}} \Pi(w, \alpha)$,

$\Pi=\sum_{v=1}^{N} \zeta_{v} \ell_{i m}^{v} \ell_{j n}^{v} E_{m n o p}^{v} \ell_{k o}^{v} \ell_{l p}^{v} \int_{\omega} w_{k l} w_{i j} \mathrm{~d} \omega-\int_{\omega} q(x, y) w(x, y) \mathrm{d} \omega$ where $E_{\text {mпор }}^{v}$ is the elasticity tensor of $v$-th orthotropic ply and

$$
\left\{\ell_{i k}^{v}\right\}_{i \mid k}=\left(\begin{array}{cc}
\cos \alpha_{v} & \sin \alpha_{v} \\
-\sin \alpha_{v} & \cos \alpha_{v}
\end{array}\right)
$$

is the tensor of transformation from the local coordinate system of the $v$-th ply into the global coordinate system.

It is not worthwhile to present the way of constructing this relation.

\section{The way of resolving}

In this work the method of alternating fulfilment of necessary conditions was used:

$$
\begin{aligned}
\sum_{v=1}^{N} \sum_{\xi, \eta=1}^{\infty} \zeta_{v}\left(P_{1 v}^{\varepsilon \rho \xi \eta} \cdot c_{v}^{4}+P_{2 v}^{\varepsilon \rho \xi \eta} \cdot c_{v}^{3} s_{v}+P_{3 v}^{\varepsilon \rho \xi \eta} \cdot c_{v}^{2} s_{v}^{2}+\right. \\
\left.\quad+P_{4 v}^{\varepsilon \rho \xi \eta} \cdot c_{v} s_{v}^{3}+P_{5 v}^{\varepsilon \rho \xi \eta} \cdot s_{v}^{4}\right) w^{\xi \eta}=q_{\varepsilon \rho} \quad(\varepsilon \rho=1,2, \ldots),
\end{aligned}
$$

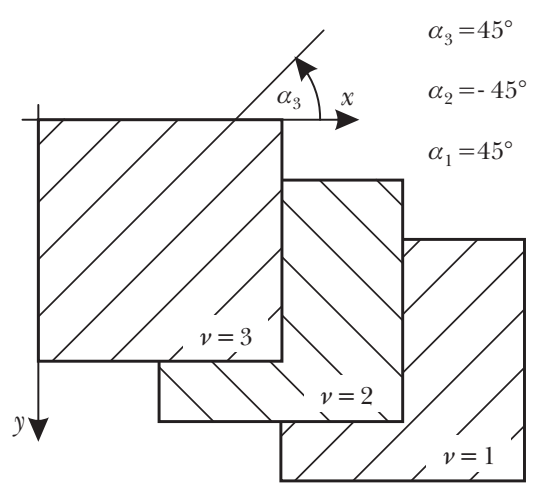

First variant

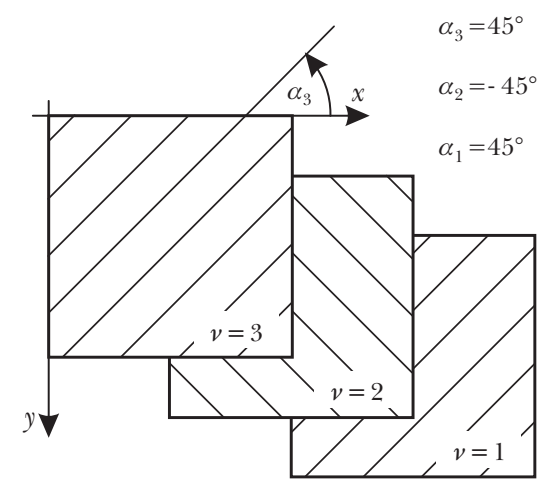

Second variant

$$
\begin{aligned}
& \sum_{\kappa, \gamma=1}^{K} \sum_{\xi, \eta=1}^{K} w^{\kappa \gamma} w^{\xi \eta}\left(R_{1 v}^{\kappa \gamma \xi \eta}+R_{2 v}^{\kappa \gamma \xi \eta} \operatorname{tg} \alpha_{v}+R_{3 v}^{\kappa \gamma \xi \eta} \operatorname{tg}^{2} \alpha_{v}+\right. \\
& \left.+R_{4 v}^{\kappa \gamma \xi \eta} \operatorname{tg}^{3} \alpha_{v}+R_{5 v}^{\kappa \gamma \xi \eta} \operatorname{tg}^{4} \alpha_{v}\right)=0 \quad(v=1,2, \ldots, N)
\end{aligned}
$$

In this $P_{\alpha v}^{\kappa \gamma \xi \eta}, R_{\alpha v}^{\kappa \gamma \xi \eta}, \zeta$ are known numbers, $c_{v}=\cos \left(\alpha_{v}\right)$, $s_{v}=\sin \left(\alpha_{v}\right)$ and $\alpha_{v}$ are the searched ply orientations.

\section{Results}

First example:

- a square plate,

- six plies that are laid symmetrically with respect to the middle plane of the plate,

- continuous loading $q=x y\left[\mathrm{~N} / \mathrm{mm}^{2}\right]$.

Graphic representation of the stiffness maximizing ply orientation (first example - Fig. 1).

The previous three solution variants have the same value as our measure of stiffness. This value of the measure of stiffness is the maximum of all values. It is interesting that the first variant is balanced and therefore not twisted. The same holds for the folowing three solution variants.

Second example:

- a rectangular plate with side ratio 1:2,

- six plies that are laid symmetrically with respect to the middle plane of the of the plate,

- continuous loading of the plate $q=x y\left[\mathrm{~N} / \mathrm{mm}^{2}\right]$.

Graphic representation of the stiffness maximizing ply orientation (second example - Fig. 2).

Fig. 1: First example

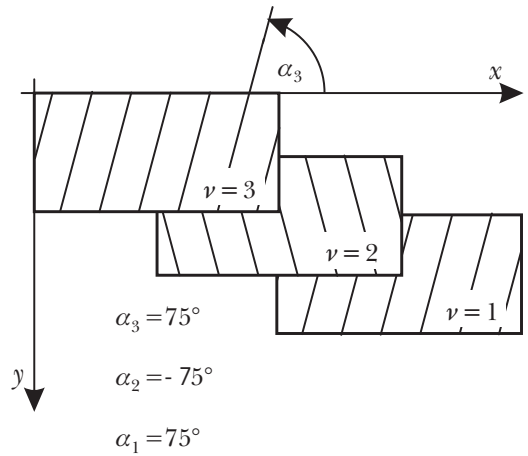

First variant

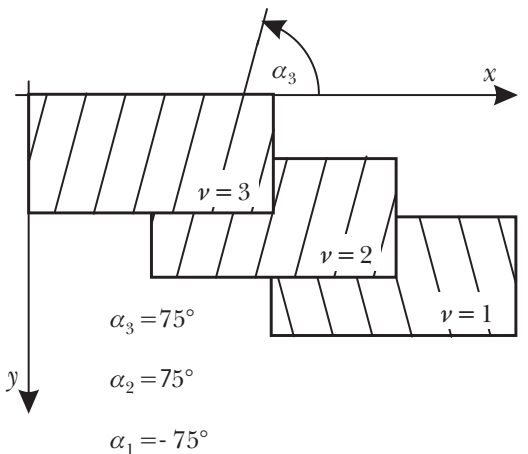

Second variant

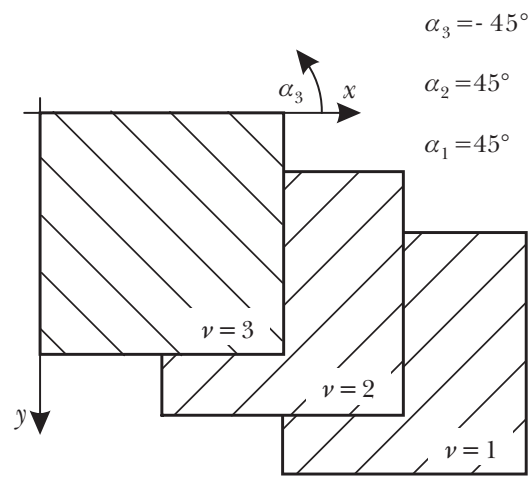

Third variant

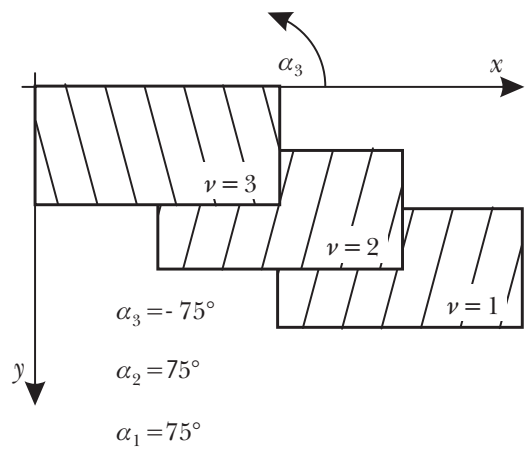

Third variant

Fig. 2: Second example 


\section{Conclusions}

We contemplate the problem of a rectangular laminate plate with common but fixed given dimensions, number of plies, thickness of plies, mechanical properties of orthotropic plies and lateral loading that is simply supported on its boundary with the aim of specifying an orientation of its plies that with maximize the measure of stiffness, which is the inverse of the measure of compliance. The measure of compliance is given by the scalar product of the deflection function describing the deformed middle plane of the plate and the lateral loading over the projection of the plate into middle plane.

We must search an actual deformed state with respect to the common orientation of the plies. This solution is used in the above expressed measure of compliance, and the minimum of this expression is searched.

It is not worthwhile to present the way of constructing of this relation. It is also not worthwhile to present the way of resolving it. Therefore, we introduce only the result.

First example: a square plate with six plies that are laid symmetrically with respect to the middle plane, of the plate. This plate is continuously loaded with the value increasing as the coordinates increase.

There are three solution variants that have the same value as our measure of stiffness. This measure of stiffness value is the maximum of all values. The optimal ply orientation is $45^{\circ}$ for all plies, the only difference is the orientation (plus, minus). It is interesting that the first variant is balanced and therefore not twisted.

Second example: a rectangular plate with side ratio 1:2 with six plies that are laid symmetrically with whit respect to the middle plane of the plate. This plate is also continuously loaded with value increasing as the coordinates increase. The same remark as for the previous example also holds for this example. The only contrast is the optimal orientation of the plies. In this example it is $75^{\circ}$.

\section{Acknowledgment}

This work has been supported by the Grant Agency of Czech Republic under contract No. 106/01/0958.

\section{References}

[1] Alexejev V. M., Tichomirov V. M., Fomin S. V.: Matematická teorie optimálních procesů. Praha: Academia 1991.

[2] Allaire G.: Shape optimization by the Homogenization Method. New York: Springer-Verlag, 2002.

[3] Gürdal Z., Haftka R. T., Hajela P.: Design and Optimization of Laminated Composite Materials. New York: John Wiley \& Sons, 1999.

[4] Washizu K.: Variational Methods in Elasticity and Plasticity. 2nd ed. Oxford: Pergamon Press, 1975. (In Russian: Variacionnyje metody v teorii uprugosti i plastičnosti. Moskva: Mir 1987.)

[5] Wilde D. J.: Globally Optimal Design. New York: John Wiley \& Sons, 1978.

Ing. Tomáš Mareš

phone:+420224352525

e-mail:marest@sgi.fsid.cvut.cz

Department of Mechanics

Division of Strength of Materials

Czech Technical University in Prague

Faculty of Mechanical Engineering

Technická 4

16607 Prague 6, Czech Republic 